\title{
ABELIAN $p$-GROUP ACTIONS ON HOMOLOGY SPHERES
}

\author{
RONALD M. DOTZEL
}

\begin{abstract}
The Borel formula is extended to an identity covering actions of arbitrary Abelian p-groups. Specifically, suppose $G$ is an Abelian p-group which acts on a finite $C W$-complex $X$ which is a $Z_{p}$-homology $n$-sphere. Each $X^{H}$ must be a $Z_{p}$-homology $n(H)$-sphere and then

$$
n-n(G)=\sum(n(K)-n(K / p))
$$

where the sum is over $A_{0}=\{K \mid G / K$ is cyclic $\}$ and the group $K / p$ is defined by

$$
K / p=\{g \in G \mid p g \in K\} \text {. }
$$

This result is an immediate corollary of Theorem 2, whose converse Theorem 1, is also proven. Thus actions of Abelian p-groups on homology spheres resemble linear representations.
\end{abstract}

0. Introduction. When an elementary Abelian $p$-group $G$ acts on a mod- $p$ homology $n$-sphere $X$, each fixed point set $X^{H}$ is a $Z_{p}$-homology sphere of dimension, say, $n(H)$. In [1, p. 175] it was first shown that

$$
n-n(G)=\sum(n(H)-n(G))
$$

where the sum runs over all corank 1 subgroups $H$. In [4], a converse result was established.

In this paper, we prove that a similar identity holds for the action of any Abelian $p$-group $G$. The converse is also proven.

Before we state the two main theorems we introduce some notation, which will be used at various points throughout the paper. If $K<G$ we set $K / p=\{g \in$ $G \mid p g \in K\}$, a subgroup of $G$. Also if $H<G$ we set

$$
A_{H}=\{K<G \mid K>H, G / K \text { cyclic }\},
$$

so that, e.g. $A_{0}=$ all subgroups with cyclic quotient $\}$. We can now state the main results.

TheOREM 1. Let $G$ be any finite Abelian p-group acting cellularly on a finite $C W$-complex $X$ such that for $H \leqslant G, H \neq 0, X^{H}$ is a $Z_{p}$-homology $n(H)$-sphere. Assume that there exists $n>0$ so that $\tilde{H}_{*}\left(X ; Z_{p}\right)=0, * \neq n$. For any subgroup $H$ of $G$ (including 0$)$ assume the following identity holds for the $G / H$ action on $X^{H}$ :

$$
n(H)-n(G)=\sum(n(K)-n(K / p))
$$

with the sum running over $A_{H}$. Assume that $n(K)-n(K / p)$ is even if $p$ is 2 and $|G / K|>2$. Note that $n(0)=n$. Also assume $n-n(G)$ is even if $p$ is odd.

Then $H_{n}\left(X ; Z_{p}\right)=Z_{p}+F$, with $F$ free over $Z_{p} G$.

Received by the editors January 10, 1979 and, in revised form, October 10, 1980.

AMS (MOS) subject classifications (1970). Primary 55C35; Secondary 55B25.

(c) 1981 American Mathematical Society $0002-9939 / 81 / 0000-0437 / \$ 02.00$ 
REMARK. Assuming $n-n(G)$ even for $p$ odd is a restriction only when $G=Z_{p} s$.

TheORem 2. Let $G$ be a finite Abelian p-group acting cellulary on a finite $C W$-complex $X$ such that each $X^{H}$ is a $Z_{p}$-homology $n(H)$-sphere, $H \neq 0$. Suppose $\tilde{H}_{i}\left(X ; Z_{p}\right)=0, i \neq n$, and $H_{n}\left(X ; Z_{p}\right)=Z_{p}+F, F$ free over $Z_{p} G$, for some integer n. Then $n-n(G)=\Sigma(n(K)-n(K / p))$, sum over $A_{0}$.

We will indicate the proof of Theorem $i$ in $\S \mathrm{i}, i=1,2$. Since the arguments here closely parallel those of [4], in general, only those necessary changes will be noted and where convenient the reader should see [4] for more complete details.

I would like to acknowledge assistance and encouragement from Professors Glen Bredon and Gary Hamrick.

1. In this section we prove Theorem 1 , stated in the Introduction.

In order to prove Theorem 1, we construct an equivariant map $\phi$ from $X$ to an appropriate linear model $S^{n}$. This map $\phi$ will induce a $Z_{p}$-homology isomorphism

$$
\phi_{*}^{H}: H_{*}\left(X^{H} ; Z_{p}\right) \rightarrow H_{*}\left(\left(S^{n}\right)^{H} ; Z_{p}\right) \text { for all } H \neq 0 \text {, }
$$

and an epimorphism when $H=0$. Study of the mapping cone of $\phi$ yields the theorem.

The linear model we need is provided by the following lemma.

Lemma 1.1. Let $X, G$ and $n$ be as in the statement of Theorem 1. Then there is $a$ linear $G$-action on $S^{n}$ such that for $H<G$, if $\left(S^{n}\right)^{H}=S^{m}$ then $m=n(H)$ where $X^{H}$ is a $\mathbf{Z}_{p}$-homology $n(H)$-sphere.

Proof. Let $K$ be any subgroup of $G$ such that $G / K$ is cyclic and let $\phi_{K}$ be a 1-dimensional complex (real, if $|G / K|=2$ ) irreducible representation of $G / K$. For any representation $\psi, \psi^{\mathrm{m}}$ denotes $\psi+\psi+\cdots+\psi$ (m-times). If 1 denotes the trivial 1-dimensional real representation, consider the real representations,

$$
V=1^{n(G)}+\sum\left(\phi_{K}\right) \frac{(n(K)-n(K / p))}{\alpha_{K}} \quad(p=2)
$$

or

$$
V=1^{n(G)}+\sum\left(\phi_{K}\right) \frac{(n(K)-n(K / p))}{2} \quad(p \neq 2)
$$

where $\alpha_{K}=1$ if $|G / K|=2, \alpha_{K}=2$ if $|G / K|>2$, and the sums are over $A_{0}$. In any case one can check that for any $H<G$

$$
V^{H}=1^{n(G)}+\sum \phi_{K} \frac{(n(K)-n(K / p))}{\alpha_{K}} \quad(p=2)
$$

or

$$
V^{H}=1^{n(G)}+\sum \phi_{K} \frac{(n(K)-n(K / p))}{2} \quad(p \neq 2)
$$

with sums over $A_{H}$. Thus $\operatorname{dim}_{\mathrm{R}} V^{K}=n(K)$. The one-point compactification of $V$ yields the required action on $S^{n}$. 
As in [4] we will assume that each sphere $\left(S^{n}\right)^{H}$ is 1 -connected and that $X$ is a suspended $G$-space (this assumption does not affect the homological conclusion we wish to obtain).

The following two lemmas are proved essentially in [4, Lemmas 2 and 3].

LEMMA 1.2. Let $X$ be a finite $C W$-complex with an action of a p-group $P$ such that all $X^{H}(H \leqslant P, H \neq 0)$ are $Z_{p}$-acyclic. Suppose there is an integer $n>0$ so that $\tilde{H}_{i}\left(X ; Z_{p}\right)=0, i \neq n$. Then $H_{n}\left(X ; Z_{p}\right)$ is a free $Z_{p}[P]$-module.

LEMMA 1.3. Given $G, X, S^{n}$ as above, suppose $\phi: X \rightarrow S^{n}$ is a G-map which induces a $Z_{p}$-homology isomorphism $\phi^{H}$ for $H \neq 0$ and an epimorphism for $H=0$ where $\phi^{H}$ : $X^{H} \rightarrow\left(S^{n}\right)^{H}$. Then $H_{n}\left(X ; Z_{p}\right)=Z_{p}+F, F$ a free $Z_{p} G$-module.

We must now show that the map $\phi$ exists. By Lemma 4 of [4] there is a map $\phi^{G}$ : $X^{G} \rightarrow\left(S^{n}\right)^{G}$ which induces a $Z_{p}$-homology isomorphism (by standard obstruction theory and the fact that $X^{G}$ is a co-H-space) (see [4] for a proof).

Obstructions to extending $\phi^{G}$ to an equivariant map $\phi: X \rightarrow S^{n}$ lie in the groups $H_{G}^{k+1}\left(X, X^{G} ; \tilde{\omega}_{k}\left(S^{n}\right)\right)$, the equivariant classical cohomology groups defined by Bredon in [3]. Using the arguments of [4] one can show that these groups all consist of torsion prime to $p$. Since $X$ (and all its skeleta) are co- $H$-spaces, a simple procedure using cogroup addition allows one to circumvent any possible obstructions which might occur. (See proof of Lemma 4 of [4].) As in [4], one needs to establish a vanishing result similar to the one given in [4, p. 284], namely that $H_{i}\left(X^{H},\left(X_{1}\right)^{H} ; Z_{p}\right)=0$ for $i>n(H)$, where $X_{1}$ is the subcomplex of points in $X$ whose isotropy subgroup has order $p$ or greater. One proves the stronger statement that $H_{i}\left(X^{H}, \cup_{S} X^{M} ; Z_{p}\right)=0$ for $i>n(H)$, where $S$ is any collection of subgroups of $G$ which contain $H$, by Mayer-Vietoris and induction on order of $H$ and cardinality of $S$ (see [5, p. 587]).

The fact that $\phi$, once obtained, has the correct properties is Smith theory and is demonstrated in [4, Lemma 9].

2. We now wish to indicate the proof of Theorem 2, which follows the argument given for Proposition 1 of [5].

Let $k$ be the cellular dimension of $X$, construct $Y_{k-1}$, a $k$-dimensional $k$-1connected $G-C W$-complex such that

$$
\operatorname{Ext}_{Z_{p} G}^{++(k-n)}\left(H_{n}\left(X ; Z_{p}\right), Z_{p}\right)=\operatorname{Ext}_{Z_{p} G}^{s}\left(H_{k}\left(Y_{k-1} ; Z_{p}\right), Z_{p}\right)
$$

for all $s \geqslant 2$.

Another finite $G-\mathrm{CW}$ complex $\hat{Y}_{k-1}$ is defined which satisfies all the hypotheses of Theorem 1 for the integer $m=\Sigma(n(K)-n(K / p))+n(G)$ (sum over $\left.A_{0}\right)$. It can be shown (e.g. [5, p. 588]) that

$$
\operatorname{Ext}_{Z_{p} G}^{s+(k-n)}\left(H_{n}\left(X ; Z_{p}\right), Z_{p}\right)=\operatorname{Ext}_{Z_{p} G}^{s+(k-m)}\left(\left(\hat{Y}_{k-1} ; Z_{p}\right), Z_{p}\right) \text {. }
$$

It follows that $m=n$ because unless $G$ is cyclic, $G$ has nonperiodic cohomology. When $G$ is cyclic, the conclusion of Theorem 2 is trivial. This completes the discussion of the arguments establishing Theorems 1 and 2. 


\section{REFERENCES}

1. A. Borel, Seminar on transformation groups, Ann. of Math. Studies, no. 46, Princeton Univ. Press, Princeton, N.J., 1960.

2. G. Bredon, Introduction to compact transformation groups, Academic Press, New York, 1972.

3. __ Equivariant cohomology theories, Lecture Notes in Math., vol. 34, Springer-Verlag, Berlin and New York, 1967.

4. R. Dotzel, A converse to the Borel formula, Trans. Amer. Math. Soc. 250 (1979), $275-287$.

5. __ A note on the Borel formula, Proc. Amer. Math. Soc. 78 (1980), 585-589.

Department of Mathematics, University of Texas, Austin, TeXas 78712

Current address: Department of Mathematics, University of Missouri-St. Louis, St. Louis, Missouri 63121 